\title{
An Analysis of Active Attacks on Anonymity Systems
}

\author{
Tianbo $\mathrm{Lu}^{1}$, Pan $\mathrm{Gao}^{1}$, Xiaofeng $\mathrm{Du}^{2}$, Yang $\mathrm{Li}^{1}$ \\ 1School of Software Engineering, Beijing University of Posts and \\ Telecommunications, 100876, Beijing, China \\ 2School of Computer Science, Beijing University of Posts and \\ Telecommunications, 100876, Beijing, China \\ lutb@bupt.edu.cn,dianergaopan@163.com
}

\begin{abstract}
Network security in the traditional sense consists of the following four elements: secret, integrity, availability and authenticity. Recent studies have indicated that: anonymity, as an important indicator of information security. In recent years, the research shows that: anonymity is also gradually being the important indicator of information security. With the rapid development of communication technology and the Internet, people pay more and more attention to personal privacy. As a result, the research on anonymous communication system is gradually increasing. P2P network's openness and the unpredictability of malicious nodes make the network user's privacy information more vulnerable to illegal theft. The attacker can not only get the contents of the file, but also can get the identity and other related privacy information of the sender and receiver. Anonymous technology is the best way to solve the privacy protection. Therefore, it is of important theoretical significance and application value to study the anonymous technology in P2P network environment. However, with the development of anonymous technology, P2P network attack technology has become the focus of research. This paper mainly analyzes the typical active attack technology.
\end{abstract}

Keywords: anonymous communication system, the typical active attack technology

\section{Introduction}

In the field of network security, more and more researchers begin to pay more attention to the research of anonymous communication technology. In a certain sense, the study of anonymous communication technology is to study how to resist all kinds of anonymous attack whose purpose is to cancel the anonymity of the system. Therefore, the research on the technology of anonymous communication system and the survivability of the system will be helpful to the development of the anonymous communication system.

The authors have investigated the literature on the Haven Project Free site, which is related to the anonymous communication attack technology, as shown in Figure 1-1 [1] .

According to the mode of attack, attack can be divided into passive and active attacks. Passive attackers only monitor traffic and analyze related information of the sender and the receiver in the communication, while active attackers can modify the means, increase, delete and delay the data packets. For example, traffic analysis is passive attacks, and denial of service attacks is active attack. This paper mainly studies the passive attack technology in anonymous communication system. Figure 1-2 shows the classification of the passive attacks. 


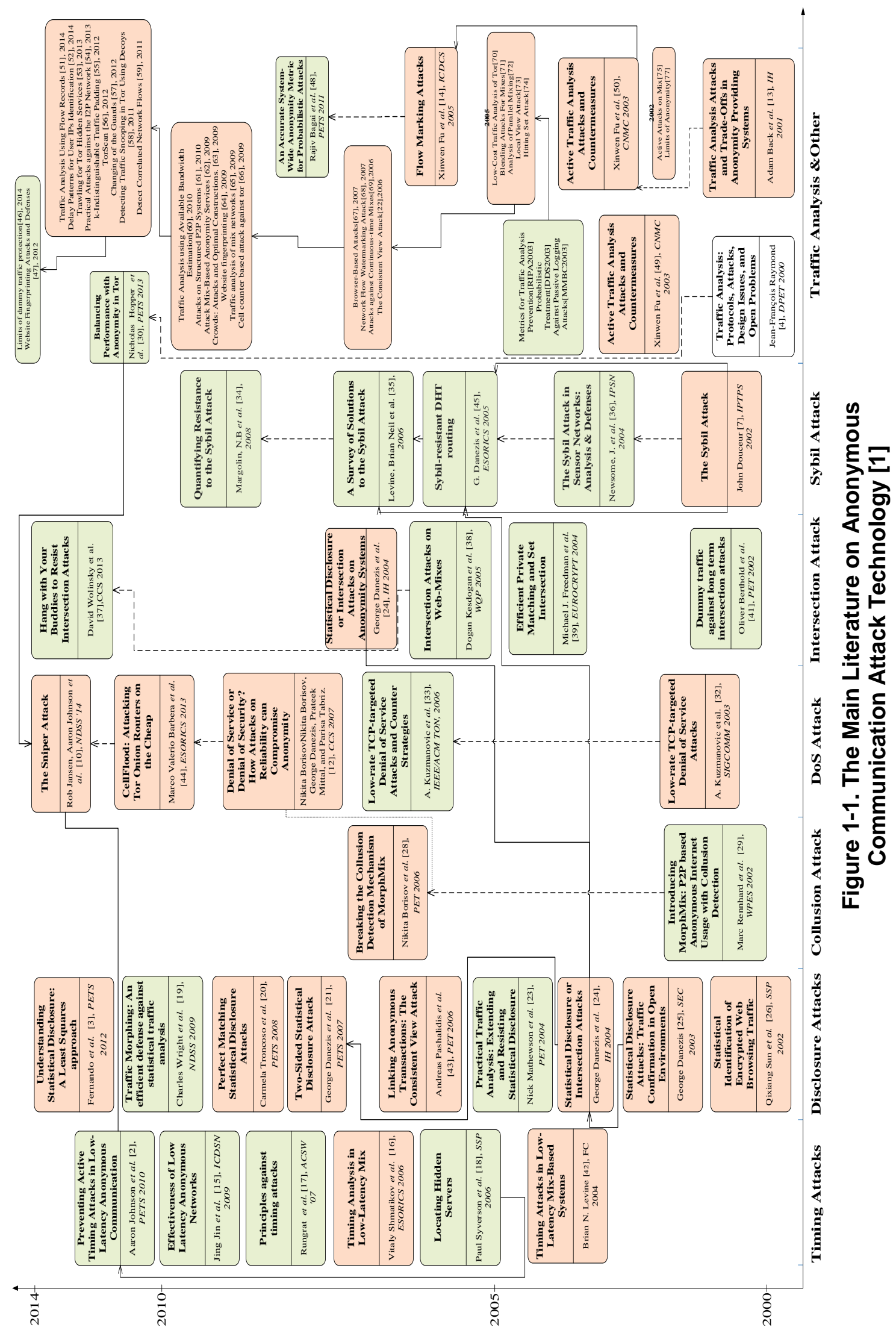




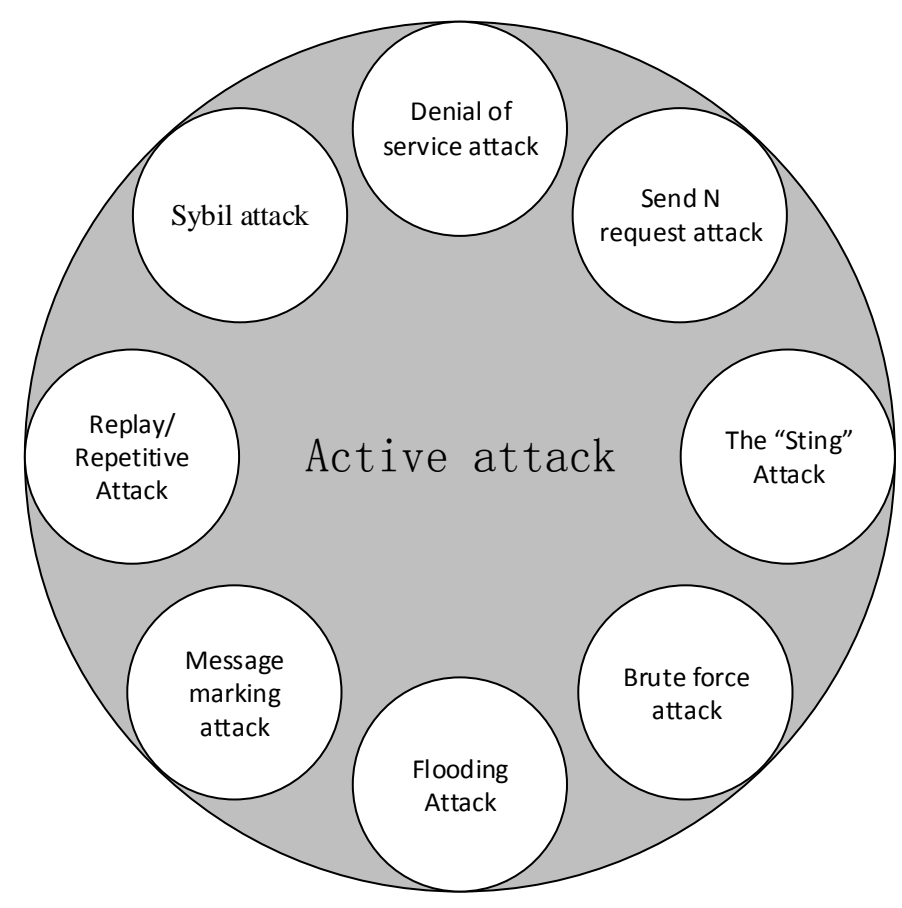

Figure 1-2. The Classification of Passive Attacks

\section{Denial of Service Attack}

Denial of service attack take up the resources of the host (including bandwidth, space and connection, etc.) by sending over a number of reasonable requests to the destination host, so that the host cannot serve for other users. The distributed denial of service attack in anonymous communication system means that the malicious nodes in the system share a large amount of data requests to other nodes, so that other nodes in the network cannot respond to the normal request, and finally make the system performance seriously reduced or crash. Figure 2-1 shows the results of the research in recent years on denial of service attacks.

Researchers from Dresden University of Technology analyze how to achieve blocking resistance for existing systems enabling anonymous web surfing and put forward the means of Defense in 2004 [2].

Nikita Borisov,Prateek Mittal from Illinois champagne, George Danezis from the University of Leuven in Belgium and Parisa Tabriz from Google analyze Denial of service attack for several anonymous communication systems and proposed the method of Selective DoS to help an attacker to destroy high latency or low latency anonymous communication systems in 2007 [3] .

The paper points out that an attacker has the choice to impact the relatively stable anonymous communication system which is very difficult to break by using a denial of service attack. DoS Selective attack method is easier than that of the whole system, and more efficient. The purpose of the attack is not to force the user to stay away from thesystem, but to reduce the reliability of the system, so it still has the function of the system. When the system is not stable, many users will try to restart the communication session, which gives the attacker to launch other attacks to provide more opportunities. The author attack and analysis the system of Tor and Mix by this attack technology. In the two case, the more nodes in the system, the more vulnerable to the DoS Selective attack. 


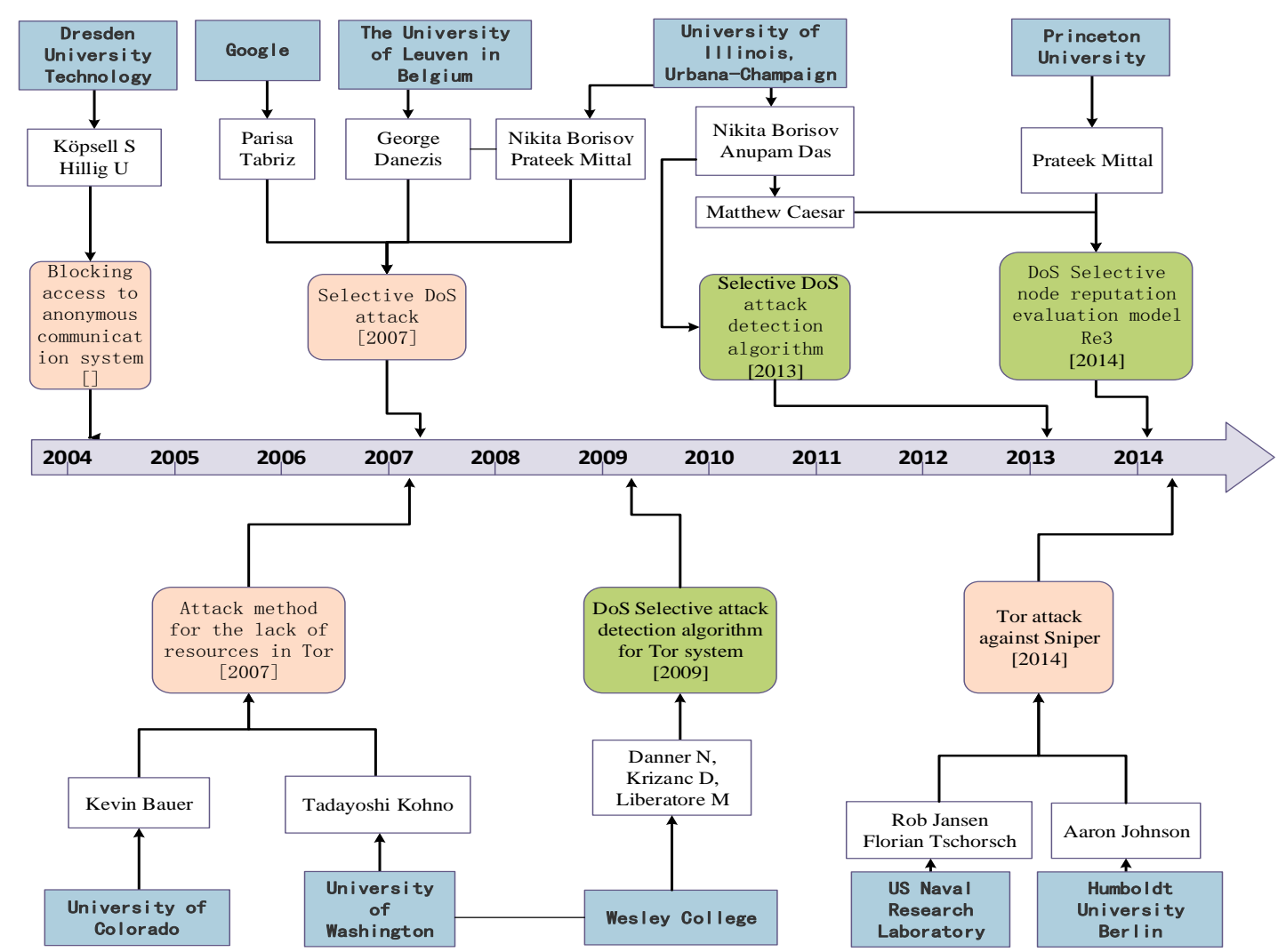

Figure 2-1. Research Situation of Denial of Service Attacks

Kevin Bauer from University of Colorado and Tadayoshi Kohno from University of Washington propose an attack method for resource lack route of Tor and prove that the method can be extended by DoS attack in 2007 [4] .

Danner from Wesleyan University propose a detection algorithm to detect Selective DoS for Tor in 2009 [5]. The algorithm basically detects each Tor node in the network, including the Tor network of the $\mathrm{N}$ participants. The complexity of detecting all the victim nodes is $\mathrm{O}(\mathrm{n})$. However, the algorithm is only based on the topology of the center.

Anupam Das and Nikita Borisov from University of Illinois, Urbana-Champaign propose a detection algorithm to protect anonymous channel for Selective DoS in 2013 [6]. This approach finds possible malicious links by detecting the actual link used.

Nikita Borisov, Matthew Caesar, Anupam Das from Illinois, Urbana-Champaign and Prateek Mittal from Princeton University proposed a node reputation evaluation model Re3 used to detect and punish relay nodes involved in active attacks such as DoS Selective in 2014 [7]. Re3 uses the exponential weighted moving average (EWMA) method, which can dynamically adjust the weighting coefficients to capture the behavior of a specific Tor relay node, and can ensure that the malicious relay nodes which are reliable and unreliable can obtain a relatively low score, and then effectively drop the active attack node based on the reputation score.

Rob Jansen, Florian Tschorsch from US Naval Research Laboratory and Aaron Johnson from Humboldt University of Berlin proposed a new denial of service attack method for Tor network, called Sniper attack [8]. The method can destroy any node of Tor. The attacker uses the reliable data transfer mechanism in the Tor system, which uses the effective protocol message to exhaust the memory of the attack node.

\section{Sybil Attack}

Sybil attack is an attack mode of P2P system. If a malicious attacker imitates multiple 
nodes in an anonymous system, it can control most of the nodes in the system, so as to destroy the $\mathrm{P} 2 \mathrm{P}$ anonymous communication system, such as routing, data redundancy, and other types of attacks. Figure 3-1 shows the results of the study of the important results of statistical exposure in recent years.

John R. Douceur from Microsoft Research Institute puts forward the concept of Sybil attack firstly in 2002 [9]. The method proposed in this paper is to prevent sybil attack from the use of trusted agents to authenticate the nodes. At the same time, the paper also points out that the Sybil attack can be realized if there is no central authority in the system.

Chris from Georgetown University and Brian Neil Levine from University of Massachusetts Amherst puts forward the method of PASID and PASID-GD to detect the Sybil attack in Ad-hoc Network in 2006 [10]. In this paper, we point out that the traffic flow can be detected in Ad-hoc Network, which can detect the Sybil attacks using a variety of network identity. This detection can be done by a single node, and the detection accuracy can be increased by using multiple trusted nodes.

Haifeng Yu from Intel Research Institute of Pittsburgh and Abraham Flaxman from Carnegie Mellon University propose the SybilGuard protocol used to limit Sybil attacks in 2006 [11]. The protocol is based on user identity "social network", and the trust relationship between the two users is represented by the side of the user's identity. Malicious users can create a lot of identity, but cannot establish a trusted relationship. Therefore, the Sybil node and the trusted node in the graph will exist in the fracture. The protocol uses this property to limit the number of malicious users to create an identity.

In order to quantify the time stability of P2P network under Sybil attack, Jochen Dinger from University of Karlsruhe proposed a new node self-registration process, to expand the P2P mechanism in 2006 [12].

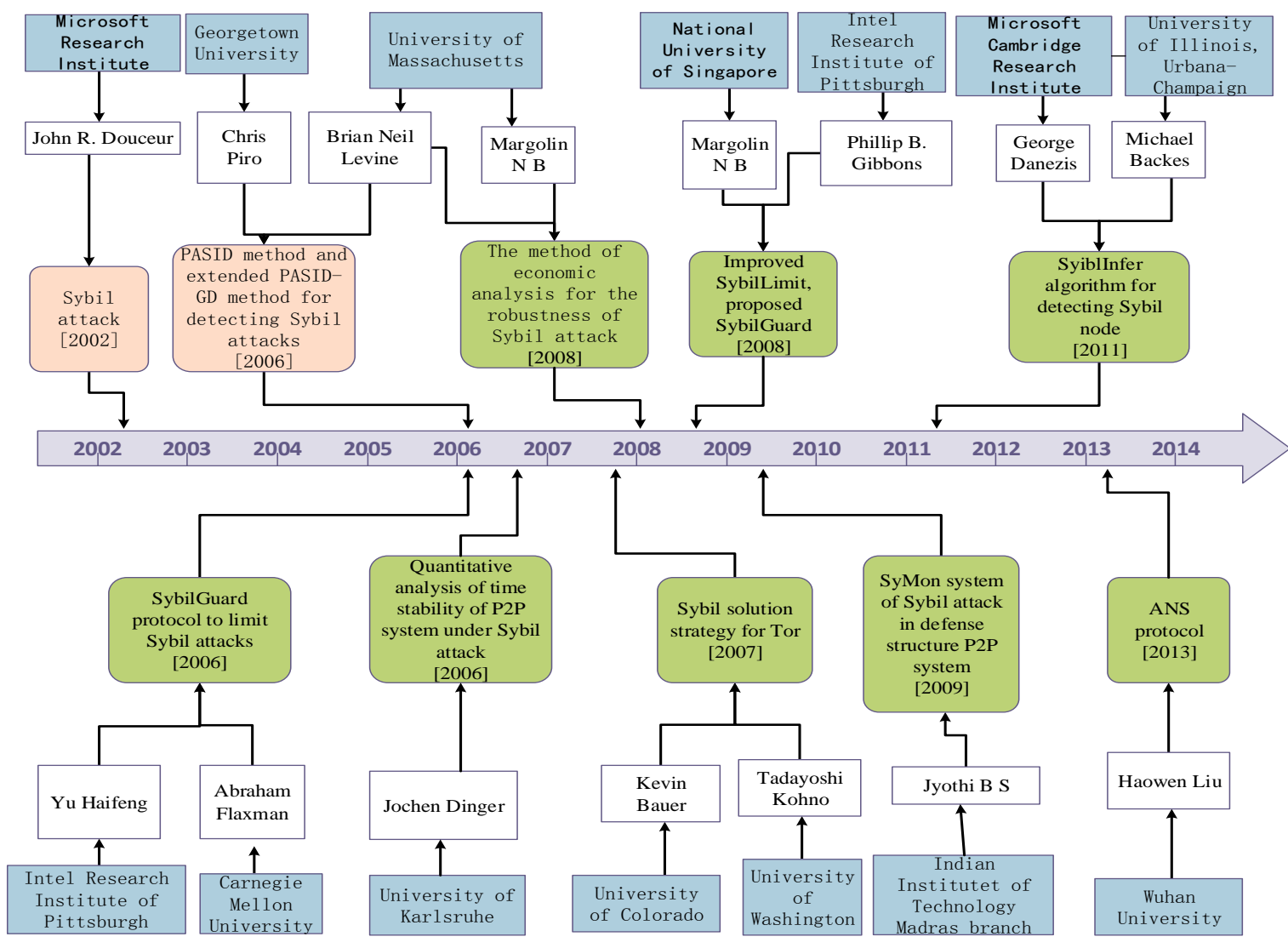

Figure 3-1. Research Situation of Sybil Attack 
Kevin Bauer from University of Colorado and Tadayoshi Kohno from University of Washington classified the Sybil attack to attack methods they proposed for Tor systems with low resource routing in 2007 [13]. In order to solve the Sybil attack, they put forward the solution strategy, that is, the directory server should limit the number of routes introduced by any single IP address, and increase the diversity of the location of the number of resources required to implement Sybil attacks.

Margolin N B and Levine B N from University of Massachusetts uses the method of economic analysis to quantitatively demonstrate the robustness of some applications and protocols against Sybil attacks, which provide a more fine-grained understanding of the attack, and allow protocol designers to measure the effectiveness of defense in 2008 [14].

Haifeng Yu from National University of Singapore and Phillip B. Gibbons from Intel Research Institute of Pittsburgh analyze the SybilGuard hypothesis and the reality gap, and put forward the SybilLimit protocol, which is similar to that of the SybilGuard protocol in 2008 [15]. They also give evidence to the basic assumption of the SybilLimit method that the agreement can be quickly mixed with a large real social network. But in fact, these two strategies are based on the heuristic, which is still not optimal.

George Danezis from Microsoft Cambridge Research Institute and Prateek Mittal from University of Illinois, Urbana-Champaign proposed a SyiblInfer algorithm using social networks to detect Sybil nodes in 2009 [16]. It can identify the nodes in the social network is an honest node or a Sybil node which is controlled by the attacker. The core of the algorithm is a probability model of an honest social network and an inference engine that can return to an area of non - honest nodes.

Jyothi B S from IIT Madras proposed the SyMon system used to defend the Sybil attack in a structured P2P system in 2009 [17]. The system associates each node with another non Sybil node (called SyMon). The SyMon of a known node is dynamically chosen, so the two nodes are all Sybil nodes with very low probability. Select the SyMon responsible for slowing down the transaction has been node related to the transaction, to make the system away from Sybil attack.

Haowen Liu from Wuhan University proposed the ANS protocol to defend against Sybil attacks [18]. This scheme is a kind of active communication strategy in Sybil attack, which is adaptive to maintain the neighbor node.

Due to the development of P2P technology, the research of Sybil attacks has been no interruption. And these studies can be directly applied to the anonymous communication system based on P2P. About the research of Sybil attacks, the focus of the research is mostly on the defense of Sybil attacks. Carnegie Mellon University, Georgetown University, University of Washington, Rheinisch Westfaelische Technische Hochschule Aachen University, Indian Institutet of Technology, and other famous universities, as well as research institutions, such as Microsoft Research Institute and other research institutions have conducted research on the attack. Our country also has the Wuhan University researchers to publish the related paper, but the result of the authority to be further verified.

The main purpose of defense against Sybil attack is to detect the dishonest node in the anonymous node. There are mainly two ways, one is to assume that any newly added nodes are likely to be malicious nodes, to strictly control and management, which can make the attacker not easy to obtain the identity of the node, so that increase the cost of attack. The second way is to tolerate some Sybil nodes and try to use the distributed network to find these nodes, and then disconnect the connection of these nodes. For this attack, the further research direction is to explore the reasonable and efficient defense method, namely, how to detect the dishonest node in the system, and how to prevent the joining of the dishonest nodes. 


\section{Other Active Attacks}

\section{a. Replay/Repetitive Attack}

Replay attack means an attacker can record the message to be traced, and then track the transmission path of the message by the method of sending the same message in Mix network.

In 2001, Oliver Berthold Pfitzmann Andreas and Ronny of the Dresden University Technology in Germany [19] analyzed the shortcomings of Free Mix, which can be the threat of replay attacks

In 2004 Gomulkiewicz M [20] of The Technological University of Wroclaw in Poland proposed a encoding design, which is based on the general purpose of the "onion like" to defend against replay attacks.

2008 Pries, R from Daktronics he State University and Fu Xinwen from the University of Massachusetts [21] put forward the replay attack method for tor system. The method assumes that the attacker controls the entry and exit nodes, and by replaying messages access the entrance note to associate the time of replay the message on entry node and the wrong time on exit node to to determine the path of anonymous communication and to destroy anonymity.

\section{b. Message marking attack}

Message making attack means that active internal attackers control the first and the last note of the message route in the anonymous communication system. Attacker make the massage on the first note to observe the exit node, such as slight modification or embedding watermark. When the same message appears, an attacker can judge the relevance of the sender and the receiver of the message. To attack the defense message marking, anonymous system must have a mechanism to remove interference information or stamp marking, reduce the tag relevance in the entrance node and the exit node.

Xinwen Fu from the University of Massachusetts proposed the flow marking attacks for flow conversion technology in the wireless anonymous communication network, and pointed out that the sign can be embedded in the radio stream by the electromagnetic interference in 2005 [22].

Xinyuan Wang from George Mason University proposed that Network Flow Watermarking Attack is used to mark the information flow in the network only. Even if the information flow is processed by any stream conversion technology and the external features are changed, the unique marking is still the same. The target reporter can track the information flow through the injection of the encoding watermark and the detection of the watermark to the information flow, thus destroying the system's anonymity [23].

\section{c. Flooding Attack}

Flooding attack is also known as n-1 attack [24]. The attacker can pick up the information of interest in the system through the flood attack. The target system of this attack usually dependent on an anonymous group, and each message in the group can only be anonymous in the group. For example, within the system of $\mathrm{N}$ users, the attacker will be forged $\mathrm{n}-1$ identity to send a message, in the communication path of the $\mathrm{N}$ message, $n-1$ is the attacker sends. Thus, an attacker can quickly rule out this n-1 message, to track the messages sent to their own.

\section{d. Brute force attack}

In the anonymous communication system, brute force attack is very similar with exhaustive attack [25]. By trying to do as much as possible to identify the relationship between the sender and the receiver, to achieve the purpose of attack. For example, in an anonymous network, an attacker can track every possible path in an anonymous network, and then obtain a set of potential message recipients. If an attacker is lucky enough, the communication relationship between the sender and the receiver can be found. But the same as the exhaustive attack, brute force attack is a very time-consuming attack and low 
efficiency. But the idea can provide guidance for the design and optimization of the anonymous communication system, such as the time and location of the flow filling.

\section{e. The "Sting" Attack}

The literature [26] refers to an active deception attack, which means that if one side of the communication is malicious, he can code in the message he send in certain conditions. For example, government agencies are looking for potential threats to create a false "Bomb production guidance website", and then find out who would like to visit the site. There are many ways to track the user's request to the site, such as changing the reply delay or sending a specific length message, etc.

In some cases, it may be more likely to compromise the user's privacy. For example, if the site provides false information about financial fraud, some users may visit the site may be put into practice, the monitoring of government agencies can arrest them.

\section{f. Send $N$ request attack}

This attack is also referred to in the literature [21]. Contrary to the "Sting" Attack that the receiver attempts to find the sender, the attack is the sender to find the receiver in this way. The kind of attack is very dangerous for non-interactive processes. An attacker sends a message that is easy to identify, and tries to confirm the information in a suspicious destination (such as a mail box).

\section{Conclusion}

In this paper, we study the technology of anonymous communication system attack. Attack technology can be divided into passive and active attacks. Passive attackers only monitor traffic and analyze related information of the sender and the receiver in the communication, while active attackers can modify the means, increase, delete and delay the data packets. This paper mainly describes the current methods of active attack, and analyzes various methods and methods of defense attack.

First of all, the background and development of anonymous communication system attack technology is introduced. As people pay more attention to the network security, anonymous communication system is studied more and more deeply and more and more researchers are devoted to the study of attack technology.

Secondly, the classification of anonymous system attack technology is introduced. This part mainly describes the current main attack mode, and analyzes the methods of these attacks, as well as the results obtained in these attacks. This part mainly describes the current main attack mode, and analyzes the methods of these attacks, as well as the results obtained in these attacks. This paper mainly analyzes Denial of service attack and Sybil attack.

In the end, the paper analyzes other active attack mode, and introduces the characteristics and the status quo of the various methods. We analyzes the advantages and disadvantages of active attack. With the development of the anonymous system, active attack technology will be further development.

\section{Acknowledgements}

This work is supported by the following programs: the National Natural Science Found ation of China under Grant No.61170273; 2010 Information Security Program of China N ational Development and Reform Commission with the title "Testing Usability and Securi ty of Network Service Software". 


\section{References}

[1] T Lu, P Yao, “Towards Attacks and Defenses of Anonymous Communication Systems," International Journal of Security and its Applications, (2015) pp. 313-328.

[2] S Köpsell, U Hillig. "How to achieve blocking resistance for existing systems enabling anonymous web surfing," Proceedings of the 2004 ACM workshop on Privacy in the electronic society. ACM, (2004) pp. 47-58.

[3] N Borisov, G Danezis, P Mittal, et al. "Denial of service or denial of security?" Proceedings of the 14th ACM conference on Computer and communications security. ACM, (2007) pp. 92-102.

[4] K Bauer, D McCoy, Grunwald, et al. "Low-resource routing attacks against tor" Proceedings of the 2007 ACM workshop on Privacy in electronic society. ACM, (2007) pp. 11-20.

[5] N Danner, D Krizanc, M Liberatore. "Detecting denial of service attacks in Tor" Financial Cryptography and Data Security. Springer Berlin Heidelberg, (2009) pp. 273-284.

[6] A Das, N Borisov. "Securing Anonymous Communication Channels under the Selective DoS Attack" Financial Cryptography and Data Security. Springer Berlin Heidelberg, (2013) pp. 362-370.

[7] A Das, N Borisov, P Mittal, et al. "Re 3: relay reliability reputation for anonymity systems"Proceedings of the 9th ACM symposium on Information, computer and communications security. ACM, (2014) pp. 63-74.

[8] R Jansen, F Tschorsch, A Johnson, et al. "The Sniper Attack: Anonymously Deanonymizing and Disabling the Tor Network" Network and Distributed Systems Security Symposium (NDSS), San Diego, CA, USA. (2014).

[9] J R Douceur. "The Sybil attack” Peer-to-peer Systems. Springer Berlin Heidelberg, (2002) pp. 251-260.

[10] C Piro, Shields C, "Levine B N. Detecting the Sybil Attack in Mobile Ad hoc Networks" SecureComm. (2006) pp. 1-11.

[11] H Yu, M Kaminsky, P B Gibbons, et al. "Sybilguard: defending against sybil attacks via social networks" ACM SIGCOMM Computer Communication Review. ACM,( 2006) pp.36(4): 267-278.

[12] J Dinger, H Hartenstein. "Defending the Sybil attack in p2p networks: Taxonomy, challenges, and a proposal for self-registration" Availability, Reliability and Security, 2006. ARES 2006. The First International Conference on. IEEE, (2006).

[13] K Bauer, D McCoy, D Grunwald, et al. "Low-resource routing attacks against tor" Proceedings of the 2007 ACM workshop on Privacy in electronic society. ACM, (2007) pp. 11-20.

[14] N B Margolin, B N Levine. "Quantifying resistance to the sybil attack" Financial Cryptography and Data Security. Springer Berlin Heidelberg, (2008) pp. 1-15.

[15] H Yu, P B Gibbons, M Kaminsky, et al. "Sybillimit: A near-optimal social network defense against sybil attacks" Security and Privacy, 2008. SP 2008. IEEE Symposium on. IEEE, (2008) pp. 3-17.

[16] G Danezis, P Mittal. "SybilInfer: Detecting Sybil Nodes using Social Networks" NDSS. (2009).

[17] B S Jyothi, D Janakiram. "SyMon: A practical approach to defend large structured P2P systems against Sybil attack" Peer-to-Peer Networking and Applications, vol. 4., no. 3, (2011), pp.289-308

[18] H Liu, F Wang. "Adaptive membership maintenance under the Sybil attack coupled with churn in P2P networks" Software Engineering and Service Science (ICSESS) (2013) pp: 545-549.

[19] O Berthold, A Pfitzmann, R Standtke. "The disadvantages of free MIX routes and how to overcome them" Designing Privacy Enhancing Technologies. Springer Berlin Heidelberg, (2001) pp. 30-45.

[20] M Gomulkiewicz, M Klonowski, M Kutylowski. "Onions based on universal re-encryption -anonymous communication immune against repetitive attack" LNCS 3325 : Proceedings of the International Workshop on Information Security Applications (WISA'04), Jeju Island, Korea,(2004) pp. 400-410.

[21] R Pries, W Yu, X Fu, et al. "A new replay attack against anonymous communication networks" Communications, 2008. ICC'08. IEEE International Conference on. IEEE, (2008) pp. 1578-1582.

[22] X Fu, Y Zhu, B Graham, et al. "On flow marking attacks in wireless anonymous communication networks" Distributed Computing Systems, 2005. ICDCS 2005. Proceedings. 25th IEEE International Conference on. IEEE, (2005) pp. 493-503.

[23] X Wang, S Chen, S Jajodia. "Network flow watermarking attack on low-latency anonymous communication systems" Security and Privacy, 2007. SP'07. IEEE Symposium on. IEEE, (2007), pp. 116-130.

[24] G. Danezis, and L Sassaman. "Heartbeat Traffic to Counter(n-1)Attacks" In Proceedings of the Workshop on Privacy in the Electronic Society(WPES2003), Washington, DC USA,(2003).

[25] J-F Raymond. "Traffic Analysis: Protocols, Attacks, Design Issues, and Open Problems" In the Proceedings of Designing Privacy Enhancing Technologies: Workshop on Design Issues in Anonymity and Unobservability, (2000) pp. 10-29. 


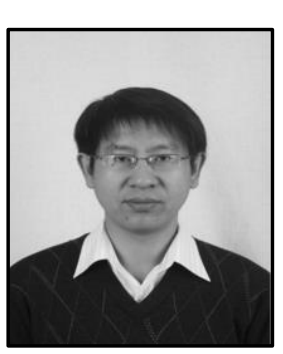

\section{Authors}

Tian-Bo Lu was born in Guizhou Province, China, 1977. He is an Associate professor in School of Software Engineering, Beijing University of Posts and Telecommunications, China. His technical interests include information security and computer network.

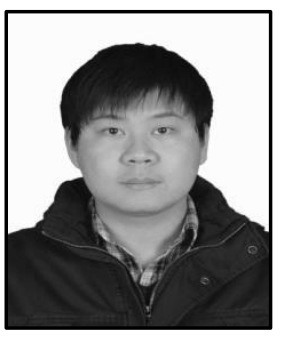

Pan Gao was born in Hebei Province, China, 1989. He is a graduate student in School of Software Engineering, Beijing University of Posts and Telecommunications, China. His technical interests include information and network security, anonymous communication.

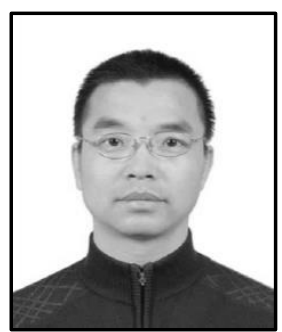

Xiao-feng Du was born in Shaanxi Province, China, 1973.He is a Lecturer in School of Computer, Beijing University of Posts and Telecommunications, China. His technical interests include information security and computer network.

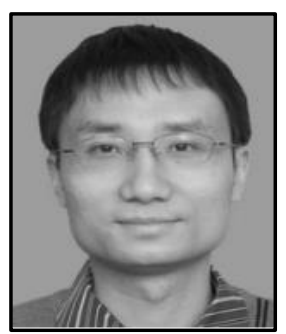

Yang Li was born in Hunan Province, China, 1978. He is a PhD and his technical interests include information security, distributed computing and $\mathrm{P} 2 \mathrm{P}$ network. 